\title{
RECOMMENDATION TO IMPROVE THE AVAILABILITY OF MEDICAL LABORATORY SERVICES IN GENERAL MEDICAL PRACTICE IN NORTHEAST BULGARIA
}

\author{
Emiliya Georgieva
}

\author{
Training and research sector "Medical laboratory assistant", Medical College, \\ Medical University of Varna
}

\begin{abstract}
In recent years in our country there are ongoing changes in the outpatient healthcare services bound up with disbalance in the territorial distribution of medical and diagnostic structures. In this study we investigated the needs and recommendations of patients, laboratory specialists and general practitioners in outpatient healthcare in Northeast Bulgaria. The data shows significant differences between the urban and rural consumption of laboratory services and recommendations are given to improve the availability associated with the construction of mobile laboratories and detect surgery in remote locations.
\end{abstract}

Keywords: recommendations, availability, medical laboratory services

\section{INTRODUCTION}

The process of aging of our population is manifested most strongly in rural areas. The biggest relative share is the population over 65 years, namely aging and regressive age structure $(5,6)$. Remote areas are served by the nearest laboratory units, but there are problems associated with remoteness, poor transport and communication difficulties in servicing (1). Defectives in these settlements are the small proportion of registered laboratories and surgery, leading to poor coordination with doctors and disrupting the integrity of the medical-diagnostic process. These circumstances require changes aimed at territori-

Address for correspondence:

Emiliya Georgieva

Medical College, Medical University of Varna

84 Tzar Osvoboditel Blvd.,

9000 Varna, Bulgaria

e-mail:emi_georgieva33@abv.bg

Received: November 5, 2015

Accepted: December 7, 2015 al distribution of laboratories in outpatient care (4). In countries where mobile diagnostic laboratories exist, they are used in order to promote and satisfy the shortcoming of medical and laboratory resources. In the US mobile laboratories were introduced to overcome these shortcomings and improve the diagnostic and treatment process of the population. The results show that these mobile laboratories work effectively to support accessibility and satisfy the need of laboratory diagnostic tests among consumers (2). The fact that in our country and the US aging population residing in rural areas is characterized by multiple chronic diseases and is economically disadvantaged indicates that the need for health services will increase. By building mobile laboratories the distance as a barrier to accessibility to this type of services will minimize (3).

\section{AIM OF THE STUDY}

The purpose of this study is to identify the needs and to analyze and summarize the recommendations of patients, laboratory specialists and general practitioners in outpatient care in Northeastern Bul- 
Emiliya Georgieva

garia to improve the availability of medical laboratory services and access to them.

\section{MATERIAL AND METODS}

The subject - respondents from Northeastern Bulgaria in the regional towns of Varna, Dobrich, Shumen and adjoining 30 municipalities divided into three groups:

1. First group - 502 patients of different gender and age. Respondents were only adults over 18 years, selected randomly immediately after contact with a medical diagnostic laboratory or a visit to a general practitioners (GP). This circumstance provides that the patient has a current and immediate impression on the activities of the medical diagnostic laboratory and GP, and can give independent answers from the GP at the time of the survey (in order to obtain the maximum possible credible answers).

2. Second group - 52 clinical laboratory doctors, registered at BMA (Bulgarian Medical Association), 63 surveyed laboratory assistants from the three areas. This covers $90 \%$ of all laboratory professionals. The survey was conducted at all outpatient laboratories in the districts of Varna, Dobrich and Shumen.

3. Third group - 389 GPs working in individual or group practices in the regions of Varna, Dobrich and Shumen. The distribution of GPs among the three regions was as follows: Varna -262 , Dobrich -69 and Shumen - 58. This covers $64 \%$ of all GPs. Most numerous were the representatives of the Varna region due to the concentration of population. In the other areas and smaller municipalities GPs with similar interests are significantly less.

We studied the opinions of 1039 respondents patients, general practitioners, laboratory assistants and doctors working in outpatient care in Northeast Bulgaria in the period July - September 2015 through a direct individual anonymous survey on the availability and accessibility of medical diagnostic laboratories in outpatient care. We used a documentary, statistical and sociological method in the study.

The documentary method was used in the analysis of literary sources in terms of availability of laboratory services in outpatient care directly related to the research problem.
Through statistical methods we interpreted the data in order to uncover the essence of observed phenomena and their interdependencies:

* Correlation analysis to determine the strength of existing relationships depending on the range of the correlation coefficient that $0<\mathrm{r}<1$ and, depending on whether the sign is reversed or not, and whether it was statistically significant at $\mathrm{p}<0.05$.

* Non-parametric analysis - taking into account distributions different from normal, as well as performing statistical analysis of complex combinational tables, opening relations and concordance criteria of Pearson (X2) which also defined the hypotheses assuming availability relation at $\mathrm{p}<0.05$.

* Parametric analysis - a comparison of the average in two independent samples.

* Graphical analysis - to visualize the observed processes and phenomena illustrating existing relationships and dependencies (volume, linear, sectoral and bar graph). MS Excel 2010 was used.

IBM SPSS Statistics version 19 (Statistical Package for Social Science) was used to process the data for quantitative analysis. The statistical difference of the indicators were evaluated with a critical significance level of $\mathrm{P}<0.05$, i.e. $95 \%$ confidence level.

\section{RESULTS AND DISCUSSION}

From the analysis of the patients' recommendations to the laboratory specialists we were impressed by the fact that half of the patients (54.3\%) were satisfied with the services of the laboratory and refrain from recommendations. One half of the respondents (19\%) see an opportunity to improve the treatment of patients by laboratory professionals towards a more polite attitude, patience and attention to patients. Other recommendations (10.3\%) were associated with an explanation of results, as well as "other", including requirements such as: mandatory use of gloves, specialized in making biological material particularly in children (9.5\%); recommendation to drop the charge when visiting a medical diagnostic laboratory (4.3\%), and a recommendation to build more laboratories (4.3\%) (Fig. 1). 


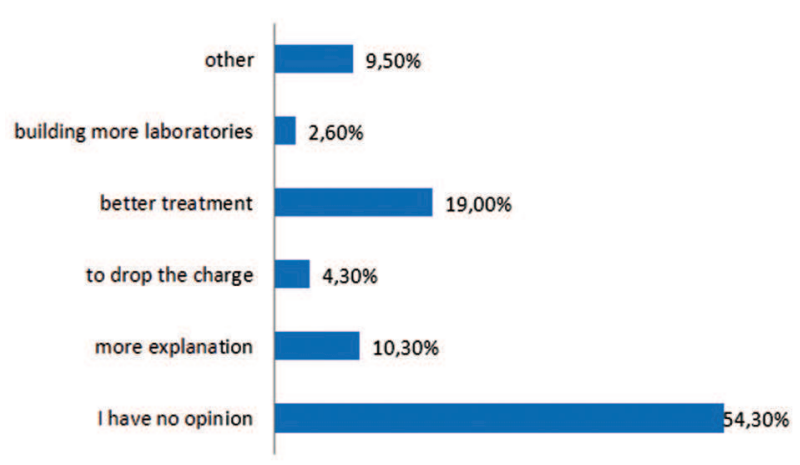

Fig. 1. Patients' recommendations to the laboratory specialists

Most patients (39.1\%) were greatly satisfied and had no recommendations to general practitioners. Those recommendations feature the willingness to release more directions for laboratory examinations 19.5\%. Respondents who believed that they needed more explanation and clarification from their doctor were only $10.2 \%$, slightly more were those who recommended to improve the treatment of patients $10.9 \%$. The recommendations of patients who answered with "other" (7.8\%) are related to financially uncertain patients and better communication with the laboratories. Another group of recommendations were about keeping the working schedule and time (2.3\%) with the aim to decrease waiting patients (3.9\%) and to reduce visiting taxes (Fig. 2).

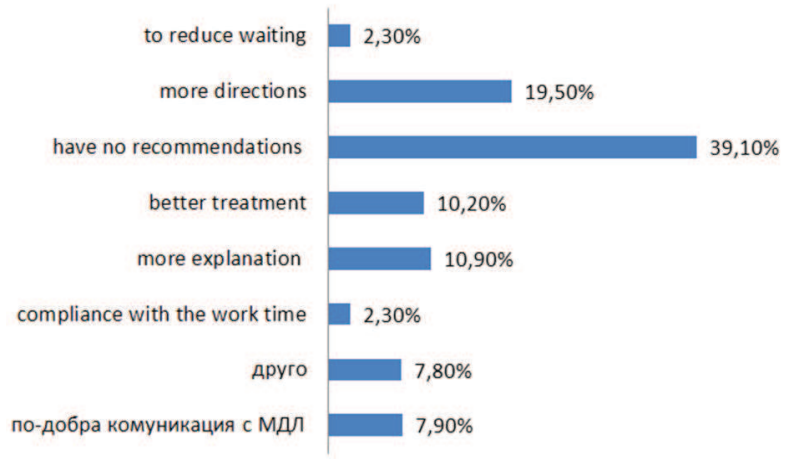

Fig. 2. Patients' recommendations to GPs

The survey found that more than half of the patients (59.8\%) were satisfied with the overall organization of the provision of laboratory services and had no recommendations. A quarter (15.6\%) believed that there is a need to build more laboratories in smaller towns and villages. Other recommendations were related to easier access to laboratory examina- tions regulated by visits of mobile laboratories once a week (4.1\%). An equal share of recommendations were associated with polite respect and more attention to patients (4.1\%), and explanation of the results (4.1\%). "Other" recommendations included: increasing the number of specialists; optimization of working time and reducing the queues in front of the offices (9\%) and optimizing examination time (3.3\%) (Fig. 3).

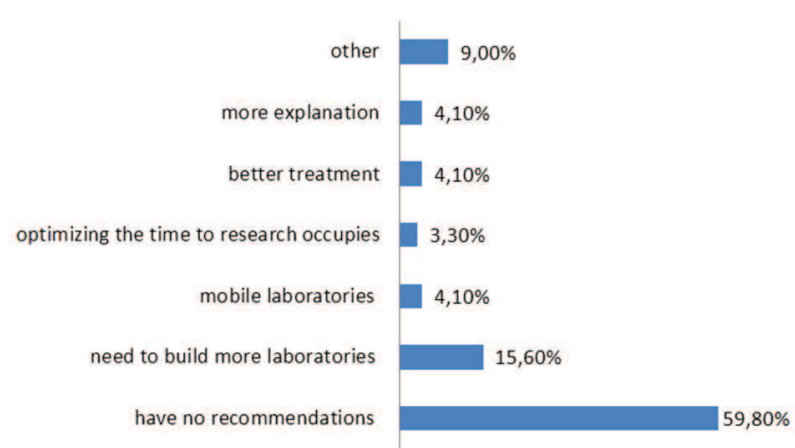

Fig. 3. Patients' recommendations in terms of the overall organization of the medical diagnostic laboratory in outpatient care

Laboratory specialists' recommendations to general practitioners were directed to more detailed explanations and instructions for patient preparation for the preliminary laboratory tests (46.8\%). With an equal share $(16.4 \%)$ were the recommendations for compliance with laboratory requirements. (the time for taking biological material, preserving the food and medicated regime and others, and a more reliable interpretation of the results). Other recommendations included improving the communication and coordination between the units (7.8\%). The proposal for registration of an electronic platform that will facilitate the access to results for all units was made only by $6.5 \%$ of the respondents. With equal shares were the recommendations for training seminars for doctors and the newest developments in the laboratory process, and for GPs to be more precise and clear in their requirements for research (3.9\%), only (2.6\%) had no recommendations (Fig. 4).

The analysis of the surveyed data showed that a significant proportion of GPs (72.7\%) believed that creating mobile laboratory teams to work on a schedule with a GP would improve patient access to laboratory services and help the healing process. A small- 


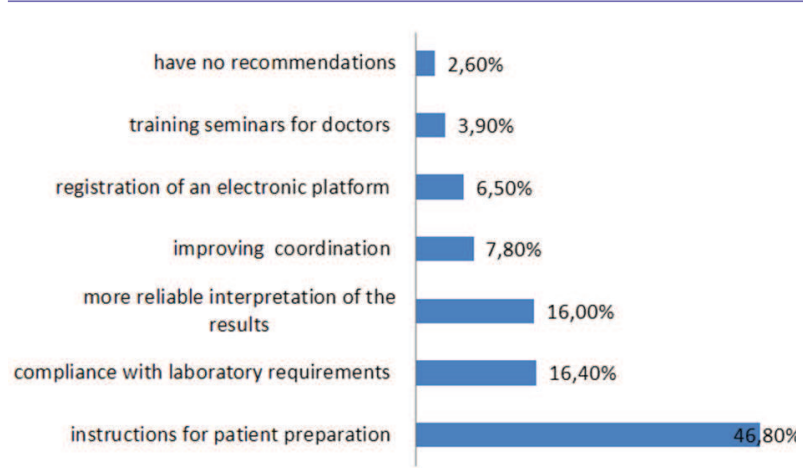

Fig. 4. Recommendations of the laboratory specialists to GPs

er part believed that improvement would be achieved by opening surgery laboratories in more settlements (23.1\%), followed by doctors who believed that there were enough laboratories (4.2\%) and there was need to take action to improve the services to the public (Fig. 5).

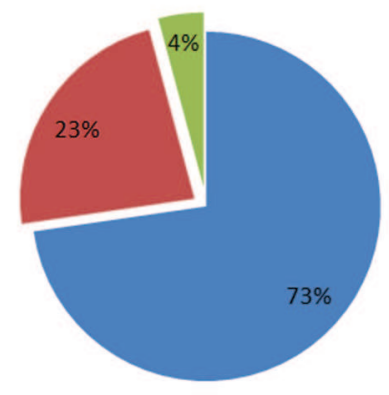

$$
\begin{aligned}
& \text { mobile laboratories } \\
& \text { mevealing surgeries } \\
& \text { no need of more } \\
& \text { laboratories }
\end{aligned}
$$

Fig. 5. Recommendations for GPs to improve laboratory services

$30.4 \%$ of the surveyed GPs had recommendations to laboratory professionals. Most GPs were of the opinion that it was most appropriate to introduce a common electronic system to facilitate the delivery of the results to doctors and patients and improve the interaction between specialists. Concerning the "other" recommendations: $25 \%$ contained answers such as: limiting the time for blood sampling (to keep medical standards intact, when a transport to more distant cities is indeed); and more responsive laboratory specialists in the absence of medical direction. A third gave recommendations for better coordination and communication between units, which consisted $13.9 \%$ of the answers. Doctors who had no recommendations were also 13.9 percent. $8.3 \%$ of the GPs expressed the opinion that the organization of surgery or mobile groups and laboratories in villages and small towns improve their work. An equal number of them (8.3\%) believed that it would be appropriate to improve the qualifications of laboratory professionals through postgraduate courses (Fig. 6).

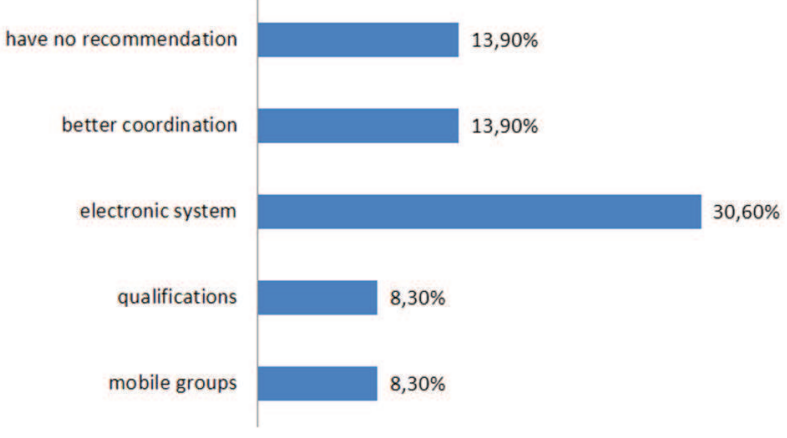

Fig. 6. Recommendations for GPs to laboratory professionals

The recommendations made by the respondents in the survey cover mainly: poor availability of medical laboratory services and specialized patient care in smaller settlements; need for qualification of the health care personnel in Northeastern Bulgaria. There is a trend for increasing patients' frustration in smaller towns with growing needs, requirements and expectations.

\section{CONCLUSION}

From the analysis of the recommendations we can conclude that the creation of mobile laboratories to improve the access to medical diagnostic laboratories or surgery in remote and inaccessible places will meet the needs of the population. Mobile teams and equipment will allow to successfully treat patients in remote areas and will support the work of doctors in outpatient care. For each health region it is possible to design a schedule of services for patients, which will be consistent with the number and needs of the patients in the local community and the work schedule of the medical diagnostic laboratory.

\section{REFERENCES}

1. Georgieva L. Availability of medical diagnostic laboratory units in Varna Region. Varna Medical Forum. 2014;3(Suppl 4);386:57-6.

2. Jia G, Yang P, Zhou J, Zhang H, Lin C, Chen J, Cai G, Yan J, Ning G. A framework design for 
the Health system for self-management promotion. Biomed Mater Eng. 2015 Aug 17;26 Suppl 1:S1731-40.

3. Mallow JA, Theeke LA, Long DM, Whetsel T, Theeke E, Mallow BK. Study protocol: mobile improvement of self-management ability through rural technology (mI SMART). Springerplus. 2015 Aug 16;4:423.

4. Tsankova G, Kaludova V, Ermenlieva N. Patients' satisfaction with laboratory services at selected medical - diagnostic laboratories in Varna. Journal of IMAB - Annual Proceeding (Scientific Papers);2014;20(2).

5. http://www.bscl.eu/docs/dokladi_cl2007.pdf

6. http://www.nsi.bg 